\title{
Wide range equation of state for fluid hydrogen within density functional theory
}

\author{
Cong Wang ${ }^{1,2}$ and Ping Zhang ${ }^{1,2, *}$ \\ ${ }^{1}$ Institute of Applied Physics and Computational Mathematics, \\ P.O. Box 8009, Beijing 100088, People's Republic of China \\ ${ }^{2}$ Center for Applied Physics and Technology, \\ Peking University, Beijing 100871, People's Republic of China
}

\begin{abstract}
Wide range equation of state (EOS) for liquid hydrogen is ultimately built by combining two kinds of density functional theory (DFT) molecular dynamics simulations, namely, first-principles molecular dynamics simulations and orbital-free molecular dynamics simulations. Specially, the present introduction of short cutoff radius pseudopotentials enables the hydrogen EOS to be available in the range $9.82 \times 10^{-4}$ to $1.347 \times 10^{3} \mathrm{~g} / \mathrm{cm}^{3}$ and up to $5 \times 10^{7} \mathrm{~K}$. By comprehensively comparing with various attainable experimental and theoretical data, we derive the conclusion that our DFT-EOS can be readily and reliably conducted to hydrodynamic simulations of the inertial confinement fusion.
\end{abstract}

PACS numbers: 31.15.A.-, 51.30.+i, 64.30.-t

\footnotetext{
${ }^{*}$ Corresponding author: zhang_ping@iapcm.ac.cn
} 


\section{INTRODUCTION}

Wide range equation of state (EOS) for hydrogen or its isotopes is of crucial interest for inertial confinement fusion (ICF) and astrophysics [1-3]. In the traditional central-hot-spot ignition designs of ICF, a deuterium-tritium (D-T) capsule is assumed to be imploded to high density either directly by high power laser pulses [4] or indirectly by X rays generated in the hohlraum [5]. Due to the fact that the compressibility of the capsule is determined by EOS, high precision EOS of the D-T fuel is essential for hydrodynamic simulations and ignition facility designs. In astrophysics, the giant Jovian planets, such as Jupiter, Saturn, Uranus, and Neptune, are composed primarily of hydrogen and helium. The knowledge on the size and mass distribution of giant Jovian planets are sensitive to the EOS of hydrogen in a wide range [6, 7]].

The EOS of hydrogen has been probed through gas gun [8], converging explosive [9], magnetically driven flyer [10], and high power laser-driven experiments [11-13], where a pressure-temperature thermodynamical domain with amplitudes of megabar (Mbar) and electron volt $(\mathrm{eV})$ has been reached. Theoretical approximations, such as classical molecular dynamics based on interatomic potentials [14], linear mixing method [15], fluid variational theory (FVT) [16], path integral Monte Carlo (PIMC) [17, 18], and quantum molecular dynamics (QMD) [19, 20], have already been employed to study high-pressure behaviors of hydrogen and its isotopes. Although it has been pursued over decades, there are still some fundamental issues worth to be noticed. For instance, the first-order phase transition from molecular to atomic fluid transition is being under intense discussion, and plasma phase transition (PPT) characterized by electronic ionization still needs to be clarified [8]. High precision, wide range EOS for hydrogen are of particularly importance for hydrodynamic simulations in ICF, especially at densities from $\sim 10^{-3}$ to $10^{3} \mathrm{~g} / \mathrm{cm}^{3}$ and temperatures up to $10^{7} \mathrm{~K}$, or even higher. Currently, SESAME-EOS table [21, 22] for hydrogen describes chemical species, such as, $\mathrm{H}_{2}$ molecules, $\mathrm{H}$ atoms, and free protons and electrons based on chemical models [15, 23-25], which are only expected to work well in the weak coupling limit.

Recently, a new EOS table based on PIMC simulations has been built [26], however, PIMC results are not consistent with experiments at pressures below $50 \mathrm{GPa}$ along the Hugoniot curve [17]. As a consequence, wide range EOS for hydrogen is highly recommended to be constructed from other promising ways for comparisons and applications. 
In the present work, a combined density functional theory (DFT) method of firstprinciples molecular dynamics (FPMD) and orbital-free molecular dynamics (OFMD) has been used to construct wide range EOS for fluid hydrogen with a temperature range $10^{3} \sim 5 \times 10^{7} \mathrm{~K}$ and density range $9.82 \times 10^{-4} \sim 1.347 \times 10^{3} \mathrm{~g} / \mathrm{cm}^{3}$. In FPMD the electrons are treated quantum mechanically through finite-temperature DFT (FTDFT) with the only approximation of exchange-correlation functional. Due to the Fermi-Dirac distribution of the electronic states, at extremely high temperatures a huge number of occupational bands have to be introduced, and FPMD simulations are then restricted. As a consequence, OFMD simulations, where the electronic kinetic energy is expressed as a functional of the local electronic density and possibly of its gradient, have been adopted to avoid the limitation. The rest of this paper is organized as follows. Section II describes the computational methods with respect to FPMD and OFMD. In section III, we discuss the EOS in detail, and finally we get our conclusions in section IV.

\section{COMPUTATIONAL METHOD}

In this section, we briefly describe the basic formalism employed to explore thermodynamic properties of fluid hydrogen. That is, two basic quantum-mechanical DFT approaches, one based on Kohn-Sham (KS) formula and the other based on orbital-free method. Then the simulation parameters are presented in detail.

\section{A. First-principles molecular dynamics}

Our FPMD simulations for fluid hydrogen have been performed by using ABINIT code [27]. In these simulations, the electrons are fully quantum mechanically treated by employing a plane-wave FT-DFT description, where the electronic state occupations follow the FermiDirac distribution. The ions move classically according to the forces from the electron density and the ion-ion repulsion. We employed the NVT (canonical) ensemble, where the number of particles $N$ and the volume are fixed [28]. The system was assumed to be in local thermodynamic equilibrium with the electron and ion temperatures being equal $\left(T_{e}=T_{i}\right)$. In these calculations, the electronic temperature was been kept constant according to the Fermi-Dirac distribution, while the ionic temperature was controlled by the Nośe thermostat. 
At each step during MD simulations, a set of electronic state functions $\left\{\Psi_{i, k}(r, t)\right\}$ for each $\mathbf{k}$-point were determined within KS construction by

$$
H_{K S} \Psi_{i, k}(r, t)=\epsilon_{i, k} \Psi_{i, k}(r, t)
$$

with

$$
H_{K S}=-\frac{1}{2} \nabla^{2}+V_{e x t}+\int \frac{n\left(r^{\prime}\right)}{\left|r-r^{\prime}\right|} d r^{\prime}+v_{x c}(r)
$$

in which the four terms respectively represent the kinetic contribution, the electron-ion interaction, the Hartree contribution, and the exchange-correlation functional. The electronic density was obtained by

$$
n(r)=\sum_{i, k} f_{i, k}\left|\Psi_{i, k}(r, t)\right|^{2} .
$$

Then by applying the velocity Verlet algorithm, based on the force from interactions between ions and electrons, a new set of positions and velocities were obtained for ions.

\section{B. Orbital-free molecular dynamics}

OFMD simulations [29 31], where the kinetic energy of the electrons is treated semiclassically, have also been used to investigate the wide range EOS for fluid hydrogen under extreme conditions. The orbital-free electronic free energy can be expressed as

$$
\begin{aligned}
F_{e}(n)= & \frac{1}{\beta} \int d r\left\{n(r) \Phi(n)-\frac{2 \sqrt{2}}{3 \pi^{2} \beta^{3 / 2}} I_{3 / 2}[\Phi(n)]\right\}+\int d r h(n) \frac{|\nabla n|^{2}}{n} \\
& +F_{x c}[n]+\frac{1}{2} \iint d r d r^{\prime} \frac{n(r) n\left(r^{\prime}\right)}{\left|r-r^{\prime}\right|}+\sum_{\ell=1}^{N_{\alpha}} Z_{\ell} \int d r \frac{n(r)}{r-R_{\ell}} \\
& -\mu \int d r\left[n(r)-\sum_{\ell=1}^{N_{\alpha}} Z_{\ell}\right]
\end{aligned}
$$

where $I_{\nu}$ is the Fermi integral of order $\nu$, and the screened potential $\Phi$ is related to the electronic density by

$$
\sum_{\ell=1}^{N_{\alpha}} Z_{\ell}=\frac{\sqrt{2}}{\pi^{2} \beta^{3 / 2}} \int d r I_{1 / 2}(\Phi[n]) .
$$

The first integral in Eq. (44), which depends only on the local electronic density in the true spirit of the Hohenberg-Kohn theorem, is the well-known finite-temperature Thomas-Fermi expression [32]. The second term in Eq. (41) denotes the von Werzsäcker correction. In the present simulations we have omitted this gradient term and worked in a Thomas-Fermi-Dirac 
form using the formula proposed by Perrot [33] to deal with the kinetic-entropic part. The orbital-free procedure treats all electrons on an equal footing, albeit approximately, with no distinction between bound and ionized electrons. Except for that, the OFMD simulation procedure is similar to that of FPMD.

\section{Simulation details}

Using the above-mentioned DFT formalisms (namely, FPMD and OFMD), we aim to build a wide range DFT-EOS table of data points for liquid hydrogen with the density ranging from $9.82 \times 10^{-4}$ to $1.347 \times 10^{3} \mathrm{~g} / \mathrm{cm}^{3}$ and temperature from $10^{3}$ to $5 \times 10^{7} \mathrm{~K}$. Generally, the Coulomb liquids can be characterized by two non-dimensional parameters. That is, the ionic coupling parameter and electronic degenerate parameter. For liquid hydrogen, the former one is commonly defined as $\Gamma_{i i}=1 /\left(k_{B} T a\right)$, which presents the ratio of the mean electrostatic potential energy and the mean kinetic energy of the ions. The degeneracy parameter $\theta=T / T_{F}$ is the ratio of the temperature to the Fermi temperature $T_{F}=\left(3 / \pi^{2} n_{e}\right)^{2 / 3} / 3$. Within the FPMD formalism, the electronic states are occupied according to the Fermi-Dirac distribution. Thus, our FPMD simulations have been restricted to temperatures lower than $T_{F}(\theta<1)$ at $\rho>0.5 \mathrm{~g} / \mathrm{cm}^{3}$. For lower densities, our FPMD simulations have been performed up to a temperature of $15.682 \mathrm{eV}$. To overcome the computational cost limit, OFMD was used in the same simulated conditions (density and temperature) as those in FPMD, and explored to extend to higher temperatures. The results indicate that both of the pressure and internal energy difference are better than $2 \%$ between QMD and OFMD simulations as $\theta \sim 1$ (see Fig. 1).

In general, FPMD and OFMD simulations based on DFT have introduced pseudopotentials to reduce the computational cost and ensure the accuracy at moderate densities. However, the pseudo-core approximation fails at high densities, where the interatomic distance is comparable with or smaller than the cutoff radius of the pseudopotential, due to pressure-induced delocalization of the core electrons and the overlapping of the pseudization spheres. In order to avoid the limitations introduced by pseudopotential approximation, a Columbic pseudopotential with a cutoff radius of 0.001 a.u. has been built [34, 35]. As the energy dependence is better than $1 \%$ between projector augmented wave (PAW) potentials and Columbic potential (see Fig. 2), we explore the EOS of hydrogen into high density 

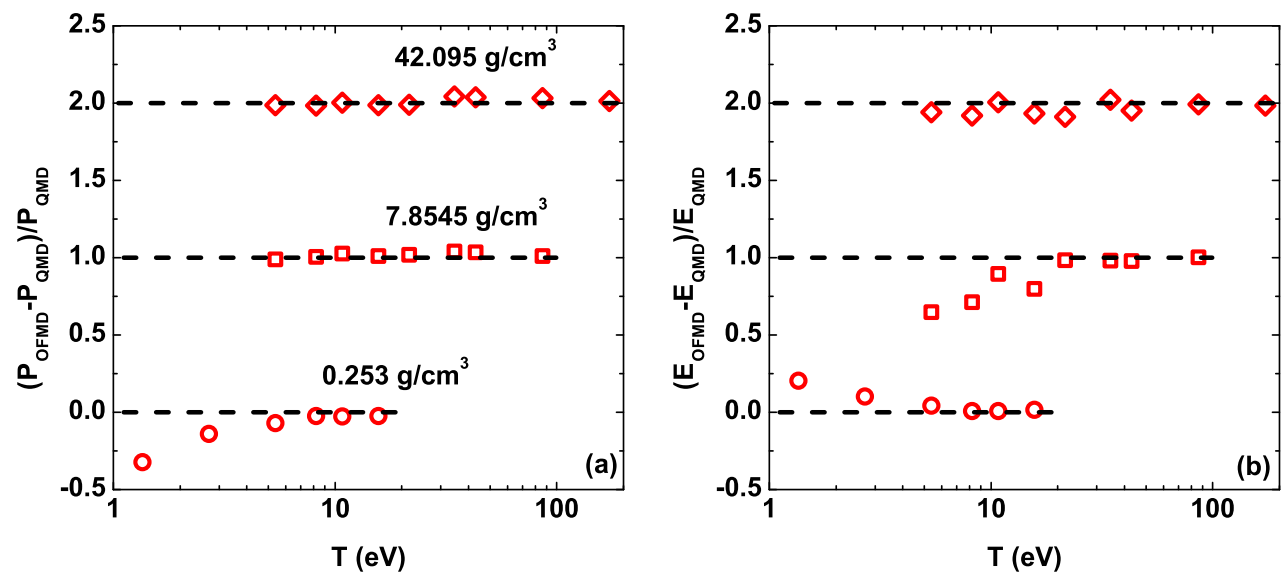

FIG. 1: (Color online) Pressure and internal energy differences between QMD and OFMD methods as functions of temperature at densities of $0.253 \mathrm{~g} / \mathrm{cm}^{3}$ (red open circles), $7.8545 \mathrm{~g} / \mathrm{cm}^{3}$ (red open squares), and $42.095 \mathrm{~g} / \mathrm{cm}^{3}$ (red open diamonds). QMD results have been plotted as the black dashed line. Each curve corresponds to an isochore. Each curve has been shifted by 1.0 from the previous one for clarity.

$\left(\sim 10^{3} \mathrm{~g} / \mathrm{cm}^{3}\right)$ by using a short cutoff radius Columbic potential.

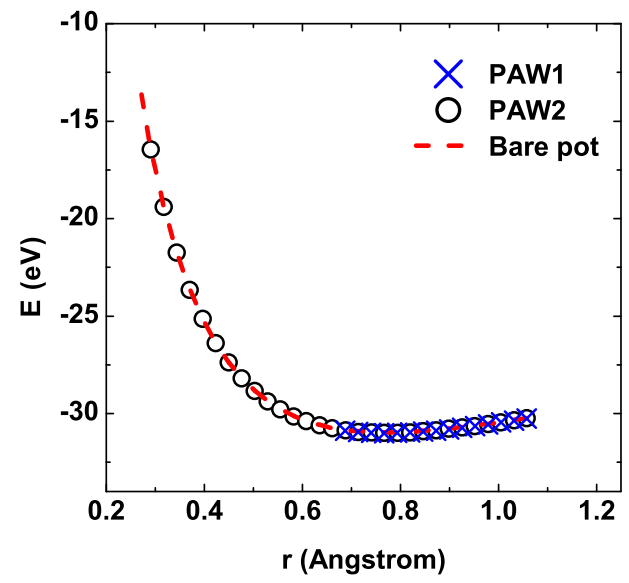

FIG. 2: (Color online) Calculated energy as a function of interatomic distance. Results are obtained from PAW potentials with a cutoff radius of 0.8 a.u. (PAW1), 0.1 a.u. (PAW2), and Columbic potential (Bare pot), as labelled in the figure.

We have considered a total number of $8 \sim 512$ atoms (corresponding to expanded and ultra dense regimes) in a series of volume-fixed supercells, which are repeated periodically throughout the space. Only $\Gamma$ point is used to sample the Brillouin zone in molecular 
dynamic simulations, because the selection of higher number of $\mathbf{k}$ points modifies the EOS within $3 \%$. Each system was assumed to be in local thermodynamic equilibrium with the electron and ion temperatures being equal $\left(T_{i}=T_{e}\right)$. In order to balance the pseudopotential approximation in the high density regime and the computational cost, two potentials have been adopted in both FPMD and OFMD simulations. That is, the PAW (with $R_{c}=0.1$ a.u.) pseudopotential $\left(\rho<30 \mathrm{~g} / \mathrm{cm}^{3}\right)$ and short cutoff radius Coulombic potential $(\rho>20$ $\left.\mathrm{g} / \mathrm{cm}^{3}\right)$, where the plane wave cutoff energy is set to $200 \mathrm{Ha}$, respectively. The exchangecorrelation functional is determined by local density approximation (LDA) with Teter-Pade parametrization [36], and the temperature dependence of exchange-correlation functional, which is convinced to be as small as negligible, is not taken into account. $\mathrm{N}_{\text {step }}=6000$ has been used in the molecular dynamic simulations, and the time steps are selected with considering different density and temperature [37]. The EOS are obtained as running average of the last 1000 steps of molecular dynamic simulations. Additionally, in FPMD simulations, sufficient electronic states have been adopted to secure the occupational number below $10^{-6}$.

\section{RESULTS AND DISCUSSION}

A wide range DFT-EOS (listed in Table I) has been constructed by data obtained from FPMD (for $\theta<1)$ and $\operatorname{OFMD}(\theta>1)$ simulations. Results are compared with previous theoretical and experimental ones in this section.

TABLE I: DFT-EOS table with pressure (GPa) and internal energy (eV/atom) for hydrogen.

\begin{tabular}{rcc}
\hline Temperature $(\mathrm{eV})$ & Pressure $(\mathrm{GPa})$ & Internal energy $(\mathrm{eV} /$ atom $)$ \\
\hline 1.348 & 0.138 & 6.290 \\
2.695 & 0.351 & 13.021 \\
5.391 & 0.864 & 26.066 \\
8.215 & 1.393 & 35.787 \\
10.781 & 1.870 & 46.596 \\
\hline
\end{tabular}

to be continued on next page 
TABLE I - continued from previous page

\begin{tabular}{|c|c|c|}
\hline Temperature(eV) & Pressure $(\mathrm{GPa})$ & Internal energy (eV/atom) \\
\hline 15.682 & 2.698 & 62.313 \\
\hline 21.563 & 3.862 & 80.142 \\
\hline 34.500 & 6.316 & 119.051 \\
\hline 43.125 & 7.950 & 144.951 \\
\hline 86.250 & 16.114 & 274.346 \\
\hline 172.500 & 32.441 & 533.100 \\
\hline 345.000 & 65.097 & 1050.593 \\
\hline 690.000 & 130.407 & 2085.570 \\
\hline 1293.750 & 244.701 & 3896.774 \\
\hline 4312.500 & 816.153 & 12952.800 \\
\hline \multicolumn{3}{|c|}{$\rho=1.5590 \times 10^{-3} \mathrm{~g} / \mathrm{cm}^{3}$} \\
\hline 1.348 & 0.212 & 6.113 \\
\hline 2.695 & 0.542 & 12.339 \\
\hline 5.391 & 1.367 & 25.339 \\
\hline 8.215 & 2.239 & 35.584 \\
\hline 10.781 & 3.084 & 45.612 \\
\hline 15.682 & 4.431 & 61.651 \\
\hline 21.563 & 6.107 & 79.572 \\
\hline 34.500 & 10.004 & 118.522 \\
\hline 43.125 & 12.598 & 144.430 \\
\hline 86.250 & 25.561 & 273.838 \\
\hline 172.500 & 51.483 & 532.598 \\
\hline 345.000 & 103.324 & 1050.092 \\
\hline 690.000 & 207.002 & 2085.071 \\
\hline 1293.750 & 388.453 & 3896.275 \\
\hline 4312.500 & 1295.667 & 12952.301 \\
\hline
\end{tabular}

to be continued on next page 
TABLE I - continued from previous page

\begin{tabular}{|c|c|c|}
\hline Temperature(eV) & Pressure(GPa) & Internal energy (eV/atom) \\
\hline \multicolumn{3}{|c|}{$\rho=2.6940 \times 10^{-3} \mathrm{~g} / \mathrm{cm}^{3}$} \\
\hline 1.348 & 0.359 & 5.927 \\
\hline 2.695 & 0.908 & 11.683 \\
\hline 5.391 & 2.298 & 24.074 \\
\hline 8.215 & 3.827 & 34.673 \\
\hline 10.781 & 5.188 & 44.264 \\
\hline 15.682 & 7.739 & 60.708 \\
\hline 21.563 & 10.493 & 78.773 \\
\hline 34.500 & 17.231 & 117.794 \\
\hline 43.125 & 21.715 & 143.715 \\
\hline 86.250 & 44.118 & 273.146 \\
\hline 172.500 & 88.912 & 531.914 \\
\hline 345.000 & 178.498 & 1049.412 \\
\hline 690.000 & 357.665 & 2084.391 \\
\hline 1293.750 & 671.210 & 3895.596 \\
\hline 4312.500 & 2238.934 & 12951.622 \\
\hline \multicolumn{3}{|c|}{$\rho=5.2620 \times 10^{-3} \mathrm{~g} / \mathrm{cm}^{3}$} \\
\hline 1.348 & 0.676 & 5.732 \\
\hline 2.695 & 1.710 & 10.860 \\
\hline 5.391 & 4.316 & 22.440 \\
\hline 8.215 & 7.278 & 33.087 \\
\hline 10.781 & 9.943 & 41.650 \\
\hline 15.682 & 14.981 & 59.245 \\
\hline 21.563 & 20.322 & 77.561 \\
\hline 34.500 & 33.495 & 116.710 \\
\hline
\end{tabular}

to be continued on next page 
TABLE I - continued from previous page

\begin{tabular}{|c|c|c|}
\hline Temperature(eV) & Pressure $(\mathrm{GPa})$ & Internal energy (eV/atom) \\
\hline 43.125 & 42.257 & 142.658 \\
\hline 86.250 & 86.019 & 272.126 \\
\hline 172.500 & 173.515 & 530.908 \\
\hline 345.000 & 348.498 & 1048.412 \\
\hline 690.000 & 698.457 & 2083.394 \\
\hline 1293.750 & 1310.879 & 3894.600 \\
\hline 4312.500 & 4373.030 & 12950.626 \\
\hline \multicolumn{3}{|c|}{$\rho=1.2473 \times 10^{-2} \mathrm{~g} / \mathrm{cm}^{3}$} \\
\hline 1.348 & 1.463 & 5.066 \\
\hline 2.695 & 3.884 & 9.320 \\
\hline 5.391 & 9.334 & 18.976 \\
\hline 8.215 & 16.559 & 30.688 \\
\hline 10.781 & 22.833 & 39.750 \\
\hline 15.682 & 34.787 & 56.895 \\
\hline 21.563 & 49.460 & 75.601 \\
\hline 34.500 & 80.720 & 115.000 \\
\hline 43.125 & 101.500 & 141.001 \\
\hline 86.250 & 204.800 & 270.546 \\
\hline 172.500 & 410.900 & 529.354 \\
\hline 345.000 & 823.100 & 1046.868 \\
\hline 690.000 & 1655.046 & 2081.854 \\
\hline 1293.750 & 3106.100 & 3893.061 \\
\hline 4312.500 & 10365.235 & 12949.087 \\
\hline \multicolumn{3}{|c|}{$\rho=2.1553 \times 10^{-2} \mathrm{~g} / \mathrm{cm}^{3}$} \\
\hline 1.348 & 2.472 & 4.493 \\
\hline
\end{tabular}

to be continued on next page 
TABLE I - continued from previous page

\begin{tabular}{|c|c|c|}
\hline Temperature $(\mathrm{eV})$ & Pressure(GPa) & Internal energy (eV/atom) \\
\hline 2.695 & 6.411 & 8.595 \\
\hline 5.391 & 16.101 & 18.019 \\
\hline 8.215 & 27.862 & 29.063 \\
\hline 10.781 & 38.592 & 37.668 \\
\hline 15.682 & 59.760 & 55.062 \\
\hline 21.563 & 84.600 & 73.978 \\
\hline 34.500 & 138.700 & 113.586 \\
\hline 43.125 & 174.600 & 139.635 \\
\hline 86.250 & 353.100 & 269.251 \\
\hline 172.500 & 709.600 & 528.081 \\
\hline 345.000 & 1425.665 & 1045.603 \\
\hline 690.000 & 2859.130 & 2080.591 \\
\hline 1293.750 & 5367.606 & 3891.798 \\
\hline 4312.500 & 17910.085 & 12947.823 \\
\hline \multicolumn{3}{|c|}{$\rho=4.2095 \times 10^{-2} \mathrm{~g} / \mathrm{cm}^{3}$} \\
\hline 1.348 & 4.264 & 4.712 \\
\hline 2.695 & 12.140 & 8.083 \\
\hline 5.391 & 30.746 & 16.663 \\
\hline 8.215 & 52.677 & 27.011 \\
\hline 10.781 & 73.257 & 35.436 \\
\hline 15.682 & 112.734 & 52.641 \\
\hline 21.563 & 162.600 & 71.692 \\
\hline 34.500 & 268.500 & 111.558 \\
\hline 43.125 & 339.100 & 137.679 \\
\hline 86.250 & 682.388 & 267.396 \\
\hline 172.500 & 1382.377 & 526.260 \\
\hline
\end{tabular}

to be continued on next page 
TABLE I - continued from previous page

\begin{tabular}{|c|c|c|}
\hline Temperature(eV) & Pressure $(\mathrm{GPa})$ & Internal energy (eV/atom) \\
\hline 345.000 & 2782.234 & 1043.791 \\
\hline 690.000 & 5581.767 & 2078.781 \\
\hline 1293.750 & 10481.126 & 3889.987 \\
\hline 4312.500 & 34977.421 & 12946.009 \\
\hline \multicolumn{3}{|c|}{$\rho=5.0 \times 10^{-2} \mathrm{~g} / \mathrm{cm}^{3}$} \\
\hline 1.348 & 5.218 & 4.035 \\
\hline 2.695 & 14.462 & 7.844 \\
\hline 5.391 & 33.584 & 17.101 \\
\hline 8.215 & 62.068 & 26.480 \\
\hline 10.781 & 86.394 & 34.845 \\
\hline 15.682 & 133.412 & 50.345 \\
\hline 21.563 & 192.000 & 71.017 \\
\hline 34.500 & 318.500 & 110.942 \\
\hline 43.125 & 401.800 & 137.082 \\
\hline 86.250 & 809.780 & 266.829 \\
\hline 172.500 & 1641.220 & 525.702 \\
\hline 345.000 & 3303.920 & 1043.235 \\
\hline 690.000 & 6629.240 & 2078.225 \\
\hline 1293.750 & 12448.600 & 3889.430 \\
\hline 4312.500 & 41545.000 & 12945.451 \\
\hline \multicolumn{3}{|c|}{$\rho=9.9781 \times 10^{-2} \mathrm{~g} / \mathrm{cm}^{3}$} \\
\hline 0.259 & 2.694 & 0.854 \\
\hline 0.431 & 3.948 & 1.153 \\
\hline 0.518 & 4.584 & 1.320 \\
\hline 0.690 & 5.537 & 1.717 \\
\hline
\end{tabular}

to be continued on next page 
TABLE I - continued from previous page

\begin{tabular}{|c|c|c|}
\hline Temperature(eV) & Pressure(GPa) & Internal energy (eV/atom) \\
\hline 0.863 & 7.924 & 1.836 \\
\hline 1.348 & 12.306 & 3.131 \\
\hline 2.695 & 28.755 & 7.031 \\
\hline 5.391 & 71.782 & 14.766 \\
\hline 8.215 & 121.424 & 23.631 \\
\hline 10.781 & 167.717 & 32.418 \\
\hline 15.682 & 260.606 & 47.720 \\
\hline 21.563 & 375.300 & 67.930 \\
\hline 34.500 & 627.300 & 107.984 \\
\hline 43.125 & 794.300 & 134.196 \\
\hline 86.250 & 1609.411 & 264.053 \\
\hline 172.500 & 3268.481 & 522.956 \\
\hline 345.000 & 6586.363 & 1040.493 \\
\hline 690.000 & 13222.325 & 2075.477 \\
\hline 1293.750 & 24835.110 & 3886.676 \\
\hline 4312.500 & 82900.032 & 12942.686 \\
\hline \multicolumn{3}{|c|}{$\rho=1.5328 \times 10^{-1} \mathrm{~g} / \mathrm{cm}^{3}$} \\
\hline 0.259 & 4.773 & 0.752 \\
\hline 0.431 & 6.561 & 1.122 \\
\hline 0.518 & 7.336 & 1.311 \\
\hline 0.690 & 9.403 & 1.733 \\
\hline 0.863 & 10.704 & 2.281 \\
\hline 1.348 & 18.789 & 3.426 \\
\hline 2.695 & 45.908 & 6.683 \\
\hline 5.391 & 110.191 & 14.078 \\
\hline 8.215 & 185.120 & 22.566 \\
\hline
\end{tabular}

to be continued on next page 
TABLE I - continued from previous page

\begin{tabular}{|c|c|c|}
\hline Temperature $(\mathrm{eV})$ & Pressure(GPa) & Internal energy (eV/atom) \\
\hline 10.781 & 253.965 & 30.910 \\
\hline 15.682 & 395.081 & 45.969 \\
\hline 21.563 & 569.300 & 65.689 \\
\hline 34.500 & 955.800 & 105.708 \\
\hline 43.125 & 1212.000 & 131.937 \\
\hline 86.250 & 2489.000 & 261.841 \\
\hline 172.500 & 5013.911 & 520.751 \\
\hline 345.000 & 10110.722 & 1038.280 \\
\hline 690.000 & 20305.244 & 2073.253 \\
\hline 1293.750 & 38145.083 & 3884.443 \\
\hline 4312.500 & 127346.277 & 12940.439 \\
\hline \multicolumn{3}{|c|}{$\rho=1.9448 \times 10^{-1} \mathrm{~g} / \mathrm{cm}^{3}$} \\
\hline 0.259 & 7.194 & 0.779 \\
\hline 0.345 & 8.599 & 0.958 \\
\hline 0.431 & 9.692 & 1.133 \\
\hline 0.518 & 10.432 & 1.363 \\
\hline 0.690 & 13.249 & 1.753 \\
\hline 0.863 & 15.283 & 2.267 \\
\hline 1.348 & 26.465 & 3.124 \\
\hline 2.695 & 60.048 & 6.458 \\
\hline 5.391 & 135.419 & 14.176 \\
\hline 8.215 & 235.860 & 21.950 \\
\hline 10.781 & 320.826 & 30.080 \\
\hline 15.682 & 499.007 & 44.982 \\
\hline 21.563 & 720.600 & 64.364 \\
\hline 34.500 & 1209.000 & 104.306 \\
\hline
\end{tabular}

to be continued on next page 
TABLE I - continued from previous page

\begin{tabular}{|c|c|c|}
\hline Temperature(eV) & Pressure(GPa) & Internal energy (eV/atom) \\
\hline 43.125 & 1537.000 & 130.534 \\
\hline 86.250 & 3158.000 & 260.445 \\
\hline 172.500 & 6396.000 & 519.350 \\
\hline 345.000 & 12849.073 & 1036.869 \\
\hline 690.000 & 25809.147 & 2071.833 \\
\hline 1293.750 & 48491.275 & 3883.014 \\
\hline 4312.500 & 161900.916 & 12938.999 \\
\hline \multicolumn{3}{|c|}{$\rho=2.5301 \times 10^{-1} \mathrm{~g} / \mathrm{cm}^{3}$} \\
\hline 0.259 & 11.871 & 0.798 \\
\hline 0.345 & 13.700 & 0.975 \\
\hline 0.431 & 15.444 & 1.162 \\
\hline 0.518 & 16.105 & 1.451 \\
\hline 0.690 & 18.874 & 1.889 \\
\hline 0.863 & 22.934 & 2.267 \\
\hline 1.348 & 36.898 & 3.296 \\
\hline 2.695 & 82.165 & 6.293 \\
\hline 5.391 & 186.413 & 13.224 \\
\hline 8.215 & 298.231 & 21.654 \\
\hline 10.781 & 414.411 & 29.201 \\
\hline 15.682 & 643.852 & 43.913 \\
\hline 21.563 & 924.300 & 62.908 \\
\hline 34.500 & 1562.000 & 102.714 \\
\hline 43.125 & 1984.000 & 128.927 \\
\hline 86.250 & 4094.000 & 258.819 \\
\hline 172.500 & 8293.000 & 517.709 \\
\hline 345.000 & 16672.990 & 1035.209 \\
\hline
\end{tabular}

to be continued on next page 
TABLE I - continued from previous page

\begin{tabular}{|c|c|c|}
\hline Temperature(eV) & Pressure(GPa) & Internal energy (eV/atom) \\
\hline 690.000 & 33498.380 & 2070.157 \\
\hline 1293.750 & 62944.837 & 3881.326 \\
\hline 4312.500 & 210176.125 & 12937.294 \\
\hline \multicolumn{3}{|c|}{$\rho=3.3676 \times 10^{-1} \mathrm{~g} / \mathrm{cm}^{3}$} \\
\hline 0.259 & 22.170 & 0.881 \\
\hline 0.345 & 24.459 & 1.064 \\
\hline 0.431 & 24.239 & 1.349 \\
\hline 0.518 & 26.790 & 1.617 \\
\hline 0.690 & 31.156 & 1.949 \\
\hline 0.863 & 37.359 & 2.291 \\
\hline 1.348 & 57.070 & 3.257 \\
\hline 2.695 & 117.535 & 6.153 \\
\hline 5.391 & 255.253 & 12.911 \\
\hline 8.215 & 414.266 & 20.725 \\
\hline 10.781 & 566.886 & 28.198 \\
\hline 15.682 & 852.768 & 42.767 \\
\hline 21.563 & 1220.000 & 61.324 \\
\hline 34.500 & 2066.000 & 100.926 \\
\hline 43.125 & 2627.000 & 127.094 \\
\hline 86.250 & 5430.000 & 256.931 \\
\hline 172.500 & 10984.218 & 515.784 \\
\hline 345.000 & 22178.636 & 1033.253 \\
\hline 690.000 & 44572.273 & 2068.174 \\
\hline 1293.750 & 83762.886 & 3879.325 \\
\hline 4312.500 & 279722.953 & 12935.268 \\
\hline
\end{tabular}

to be continued on next page 
TABLE I - continued from previous page

\begin{tabular}{|c|c|c|}
\hline Temperature(eV) & Pressure(GPa) & Internal energy (eV/atom) \\
\hline \multicolumn{3}{|c|}{$\rho=4.1867 \times 10^{-1} \mathrm{~g} / \mathrm{cm}^{3}$} \\
\hline 0.086 & 28.457 & 0.666 \\
\hline 0.259 & 36.339 & 0.954 \\
\hline 0.431 & 36.579 & 1.480 \\
\hline 0.690 & 47.351 & 2.009 \\
\hline 0.863 & 55.779 & 2.342 \\
\hline 1.348 & 81.633 & 3.291 \\
\hline 2.695 & 156.889 & 6.117 \\
\hline 5.391 & 326.268 & 12.694 \\
\hline 8.215 & 522.548 & 20.335 \\
\hline 10.781 & 685.171 & 27.621 \\
\hline 15.682 & 1058.104 & 41.926 \\
\hline 21.563 & 1515.000 & 60.154 \\
\hline 34.500 & 2562.000 & 99.553 \\
\hline 43.125 & 3267.000 & 125.671 \\
\hline 86.250 & 6744.000 & 255.430 \\
\hline 172.500 & 13690.000 & 514.238 \\
\hline 345.000 & 27562.538 & 1031.671 \\
\hline 690.000 & 55403.077 & 2066.565 \\
\hline 1293.750 & 104128.019 & 3877.696 \\
\hline 4312.500 & 347756.729 & 12933.615 \\
\hline \multicolumn{3}{|c|}{$\rho=5.0 \times 10^{-1} \mathrm{~g} / \mathrm{cm}^{3}$} \\
\hline 0.086 & 43.727 & 0.739 \\
\hline 0.259 & 49.036 & 1.148 \\
\hline 0.431 & 53.691 & 1.583 \\
\hline 0.690 & 68.576 & 2.092 \\
\hline
\end{tabular}

to be continued on next page 
TABLE I - continued from previous page

\begin{tabular}{|c|c|c|}
\hline Temperature(eV) & Pressure(GPa) & Internal energy (eV/atom) \\
\hline 0.863 & 79.054 & 2.419 \\
\hline 1.348 & 110.256 & 3.352 \\
\hline 2.695 & 203.690 & 6.226 \\
\hline 5.391 & 403.036 & 12.590 \\
\hline 8.215 & 634.218 & 20.070 \\
\hline 10.781 & 854.595 & 27.285 \\
\hline 15.682 & 1293.154 & 41.537 \\
\hline 21.563 & 1799.000 & 59.237 \\
\hline 34.500 & 3020.820 & 98.444 \\
\hline 43.125 & 3844.600 & 124.507 \\
\hline 86.250 & 7984.900 & 254.174 \\
\hline 172.500 & 16287.600 & 512.930 \\
\hline 345.000 & 32907.200 & 1030.326 \\
\hline 690.000 & 66155.400 & 2065.190 \\
\hline 1293.750 & 124347.000 & 3876.302 \\
\hline 4312.500 & 415320.000 & 12932.195 \\
\hline \multicolumn{3}{|c|}{$\rho=7.9825 \times 10^{-1} \mathrm{~g} / \mathrm{cm}^{3}$} \\
\hline 1.348 & 259.313 & 3.792 \\
\hline 2.695 & 404.291 & 6.492 \\
\hline 5.391 & 718.712 & 12.638 \\
\hline 8.215 & 1077.005 & 19.843 \\
\hline 10.781 & 1423.714 & 26.707 \\
\hline 15.682 & 2108.910 & 40.544 \\
\hline 21.563 & 2905.275 & 57.192 \\
\hline 34.500 & 4831.500 & 95.738 \\
\hline 43.125 & 6136.150 & 121.574 \\
\hline
\end{tabular}

to be continued on next page 
TABLE I - continued from previous page

\begin{tabular}{|c|c|c|}
\hline Temperature(eV) & Pressure(GPa) & Internal energy (eV/atom) \\
\hline 86.250 & 12721.700 & 250.794 \\
\hline 172.500 & 25961.000 & 509.293 \\
\hline 345.000 & 52483.001 & 1026.515 \\
\hline 690.000 & 105556.001 & 2061.257 \\
\hline 1293.750 & 198451.502 & 3872.289 \\
\hline 4312.500 & 662975.008 & 12928.083 \\
\hline \multicolumn{3}{|c|}{$\rho=9.8181 \times 10^{-1} \mathrm{~g} / \mathrm{cm}^{3}$} \\
\hline 1.348 & 384.866 & 4.176 \\
\hline 2.695 & 560.854 & 6.845 \\
\hline 5.391 & 944.470 & 12.880 \\
\hline 8.215 & 1382.305 & 19.957 \\
\hline 10.781 & 1796.059 & 26.734 \\
\hline 15.682 & 2632.108 & 40.258 \\
\hline 21.563 & 3678.614 & 57.308 \\
\hline 34.500 & 5871.477 & 93.996 \\
\hline 43.125 & 7456.522 & 120.184 \\
\hline 86.250 & 15556.243 & 252.157 \\
\hline 172.500 & 31880.886 & 512.898 \\
\hline 345.000 & 64517.773 & 1030.922 \\
\hline 690.000 & 129799.546 & 2065.866 \\
\hline 1293.750 & 244056.648 & 3876.955 \\
\hline 4312.500 & 815392.161 & 12932.766 \\
\hline \multicolumn{3}{|c|}{$\rho=1.2263 \mathrm{~g} / \mathrm{cm}^{3}$} \\
\hline 1.348 & 591.578 & 4.774 \\
\hline 2.695 & 811.125 & 7.441 \\
\hline
\end{tabular}

to be continued on next page 
TABLE I - continued from previous page

\begin{tabular}{|c|c|c|}
\hline Temperature $(\mathrm{eV})$ & Pressure(GPa) & Internal energy (eV/atom) \\
\hline 5.391 & 1282.332 & 13.347 \\
\hline 8.215 & 1816.492 & 20.228 \\
\hline 10.781 & 2324.122 & 26.844 \\
\hline 15.682 & 3353.252 & 40.091 \\
\hline 21.563 & 4638.863 & 56.811 \\
\hline 34.500 & 7391.677 & 92.514 \\
\hline 43.125 & 9309.797 & 118.716 \\
\hline 86.250 & 19393.895 & 250.119 \\
\hline 172.500 & 39770.389 & 510.828 \\
\hline 345.000 & 80525.778 & 1028.729 \\
\hline 690.000 & 162054.556 & 2063.622 \\
\hline 1293.750 & 304757.918 & 3874.666 \\
\hline 4312.500 & 1018329.725 & 12930.412 \\
\hline \multicolumn{3}{|c|}{$\rho=1.5591 \mathrm{~g} / \mathrm{cm}^{3}$} \\
\hline 2.695 & 1217.205 & 8.329 \\
\hline 5.391 & 1802.722 & 14.093 \\
\hline 8.215 & 2471.084 & 20.813 \\
\hline 10.781 & 3107.909 & 27.280 \\
\hline 15.682 & 4394.780 & 40.431 \\
\hline 21.563 & 6008.367 & 56.715 \\
\hline 34.500 & 9711.442 & 94.365 \\
\hline 43.125 & 11868.865 & 117.384 \\
\hline 86.250 & 24627.130 & 248.026 \\
\hline 172.500 & 50504.260 & 508.563 \\
\hline 345.000 & 102305.520 & 1026.416 \\
\hline 690.000 & 205947.039 & 2061.235 \\
\hline
\end{tabular}

to be continued on next page 
TABLE I - continued from previous page

\begin{tabular}{|c|c|c|}
\hline Temperature(eV) & Pressure(GPa) & Internal energy (eV/atom) \\
\hline 1293.750 & 387371.949 & 3872.216 \\
\hline 4312.500 & 1294596.497 & 12927.871 \\
\hline \multicolumn{3}{|c|}{$\rho=2.0241 \mathrm{~g} / \mathrm{cm}^{3}$} \\
\hline 2.695 & 1914.054 & 9.701 \\
\hline 5.391 & 2653.269 & 15.315 \\
\hline 8.215 & 3499.908 & 21.844 \\
\hline 10.781 & 4303.718 & 28.115 \\
\hline 15.682 & 5940.816 & 40.924 \\
\hline 21.563 & 8003.076 & 56.885 \\
\hline 34.500 & 12739.349 & 93.854 \\
\hline 43.125 & 15981.851 & 119.108 \\
\hline 86.250 & 31973.372 & 245.712 \\
\hline 172.500 & 65497.743 & 505.963 \\
\hline 345.000 & 132718.486 & 1023.755 \\
\hline 690.000 & 267252.973 & 2058.470 \\
\hline 1293.750 & 502770.574 & 3869.360 \\
\hline 4312.500 & 1680568.581 & 12924.885 \\
\hline \multicolumn{3}{|c|}{$\rho=3.6956 \mathrm{~g} / \mathrm{cm}^{3}$} \\
\hline 2.695 & 5561.156 & 15.030 \\
\hline 5.391 & 6851.582 & 20.465 \\
\hline 8.215 & 8282.303 & 26.503 \\
\hline 10.781 & 9683.735 & 32.433 \\
\hline 15.682 & 12471.118 & 44.273 \\
\hline 21.563 & 16109.020 & 59.548 \\
\hline 34.500 & 24440.344 & 94.995 \\
\hline
\end{tabular}

to be continued on next page 
TABLE I - continued from previous page

\begin{tabular}{|c|c|c|}
\hline Temperature(eV) & Pressure(GPa) & Internal energy (eV/atom) \\
\hline 43.125 & 30151.959 & 119.149 \\
\hline 86.250 & 58805.086 & 239.239 \\
\hline 172.500 & 119458.718 & 500.123 \\
\hline 345.000 & 241975.435 & 1017.331 \\
\hline 690.000 & 487450.871 & 2051.576 \\
\hline 1293.750 & 917350.383 & 3862.109 \\
\hline 4312.500 & 3067567.944 & 12917.150 \\
\hline \multicolumn{3}{|c|}{$\rho=5.0 \mathrm{~g} / \mathrm{cm}^{3}$} \\
\hline 2.695 & 9549.150 & 19.264 \\
\hline 5.391 & 11289.594 & 24.705 \\
\hline 8.215 & 13149.412 & 30.532 \\
\hline 10.781 & 14947.198 & 36.149 \\
\hline 15.682 & 18635.588 & 47.707 \\
\hline 21.563 & 23350.696 & 62.524 \\
\hline 34.500 & 33750.403 & 94.172 \\
\hline 43.125 & 41345.121 & 119.872 \\
\hline 86.250 & 80760.602 & 244.172 \\
\hline 172.500 & 162974.601 & 496.972 \\
\hline 345.000 & 329461.530 & 1026.672 \\
\hline 690.000 & 662036.697 & 2067.672 \\
\hline 1293.750 & 1243990.000 & 3869.013 \\
\hline 4312.500 & 4152780.000 & 12918.432 \\
\hline \multicolumn{3}{|c|}{$\rho=7.8545 \mathrm{~g} / \mathrm{cm}^{3}$} \\
\hline 5.391 & 23919.083 & 32.364 \\
\hline 8.215 & 26366.144 & 35.213 \\
\hline
\end{tabular}

to be continued on next page 
TABLE I - continued from previous page

\begin{tabular}{|c|c|c|}
\hline Temperature(eV) & Pressure(GPa) & Internal energy (eV/atom) \\
\hline 10.781 & 28417.191 & 40.201 \\
\hline 15.682 & 34567.155 & 50.461 \\
\hline 21.563 & 40887.110 & 63.836 \\
\hline 34.500 & 57821.094 & 96.066 \\
\hline 43.125 & 69405.461 & 118.905 \\
\hline 86.250 & 129424.016 & 240.005 \\
\hline 172.500 & 257431.789 & 492.021 \\
\hline 345.000 & 517526.963 & 968.205 \\
\hline 690.000 & 1040160.000 & 2052.492 \\
\hline 1293.750 & 1953890.000 & 3857.032 \\
\hline 4312.500 & 6522980.000 & 12881.855 \\
\hline \multicolumn{3}{|c|}{$\rho=1.2473 \times 10^{1} \mathrm{~g} / \mathrm{cm}^{3}$} \\
\hline 5.391 & 52334.071 & 45.782 \\
\hline 8.215 & 56804.007 & 50.849 \\
\hline 10.781 & 60424.429 & 54.502 \\
\hline 15.682 & 68219.429 & 62.075 \\
\hline 21.563 & 77112.745 & 74.889 \\
\hline 34.500 & 103991.965 & 105.034 \\
\hline 43.125 & 121078.467 & 127.234 \\
\hline 86.250 & 213335.193 & 245.844 \\
\hline 172.500 & 412180.825 & 487.534 \\
\hline 345.000 & 823130.585 & 1015.634 \\
\hline 690.000 & 1652820.000 & 2028.342 \\
\hline 1293.750 & 3103200.000 & 3841.490 \\
\hline 4312.500 & 10356600.000 & 12898.888 \\
\hline
\end{tabular}

to be continued on next page 
TABLE I - continued from previous page

\begin{tabular}{|c|c|c|}
\hline Temperature(eV) & Pressure(GPa) & Internal energy (eV/atom) \\
\hline \multicolumn{3}{|c|}{$\rho=2.1553 \times 10^{1} \mathrm{~g} / \mathrm{cm}^{3}$} \\
\hline 5.391 & 132965.313 & 69.516 \\
\hline 8.215 & 140631.941 & 75.069 \\
\hline 10.781 & 147606.997 & 79.707 \\
\hline 15.682 & 161508.892 & 91.188 \\
\hline 21.563 & 176692.441 & 98.682 \\
\hline 34.500 & 214260.781 & 124.801 \\
\hline 43.125 & 244807.793 & 144.762 \\
\hline 86.250 & 397368.524 & 255.511 \\
\hline 172.500 & 733042.082 & 498.257 \\
\hline 345.000 & 1436410.000 & 1015.776 \\
\hline 690.000 & 2860880.000 & 2037.937 \\
\hline 1293.750 & 5365630.000 & 3849.903 \\
\hline 4312.500 & 17899300.000 & 12890.991 \\
\hline \multicolumn{3}{|c|}{$\rho=4.2095 \times 10^{1} \mathrm{~g} / \mathrm{cm}^{3}$} \\
\hline 5.391 & 420619.812 & 120.275 \\
\hline 8.215 & 436741.892 & 121.507 \\
\hline 10.781 & 445038.475 & 125.749 \\
\hline 15.682 & 477946.889 & 134.127 \\
\hline 21.563 & 500374.227 & 144.655 \\
\hline 34.500 & 574053.275 & 169.598 \\
\hline 43.125 & 621244.176 & 187.496 \\
\hline 86.250 & 900873.051 & 288.390 \\
\hline 172.500 & 1516040.000 & 519.066 \\
\hline 345.000 & 2861940.000 & 996.990 \\
\hline 690.000 & 5626920.000 & 2054.507 \\
\hline
\end{tabular}

to be continued on next page 
TABLE I - continued from previous page

\begin{tabular}{|c|c|c|}
\hline Temperature(eV) & Pressure $(\mathrm{GPa})$ & Internal energy (eV/atom) \\
\hline 1293.750 & 10503500.000 & 3853.605 \\
\hline 4312.500 & 34970300.000 & 12898.971 \\
\hline \multicolumn{3}{|c|}{$\rho=8.4190 \times 10^{1} \mathrm{~g} / \mathrm{cm}^{3}$} \\
\hline 5.391 & 1380830.000 & 197.188 \\
\hline 8.215 & 1405720.000 & 199.217 \\
\hline 10.781 & 1437320.000 & 206.732 \\
\hline 15.682 & 1491360.000 & 217.589 \\
\hline 21.563 & 1546100.000 & 223.268 \\
\hline 34.500 & 1657160.000 & 252.920 \\
\hline 43.125 & 1765120.000 & 269.112 \\
\hline 86.250 & 2250500.000 & 359.484 \\
\hline 172.500 & 3390870.000 & 572.109 \\
\hline 345.000 & 5962050.000 & 1047.419 \\
\hline 690.000 & 11406900.000 & 2049.414 \\
\hline 1293.750 & 21127400.000 & 3824.306 \\
\hline 4312.500 & 69971100.000 & 12895.733 \\
\hline \multicolumn{3}{|c|}{$\rho=1.6838 \times 10^{2} \mathrm{~g} / \mathrm{cm}^{3}$} \\
\hline 5.391 & 4470890.000 & 348.905 \\
\hline 8.215 & 4531710.000 & 353.258 \\
\hline 10.781 & 4567730.000 & 357.249 \\
\hline 15.682 & 4706480.000 & 365.018 \\
\hline 21.563 & 4754720.000 & 374.545 \\
\hline 34.500 & 5038440.000 & 396.244 \\
\hline 43.125 & 5235950.000 & 411.271 \\
\hline 86.250 & 6139410.000 & 492.918 \\
\hline
\end{tabular}

to be continued on next page 
TABLE I - continued from previous page

\begin{tabular}{|c|c|c|}
\hline Temperature(eV) & Pressure(GPa) & Internal energy (eV/atom) \\
\hline 172.500 & 8286640.000 & 684.142 \\
\hline 345.000 & 13024000.000 & 1128.948 \\
\hline 690.000 & 23505400.000 & 2101.594 \\
\hline 1293.750 & 42790700.000 & 3685.375 \\
\hline 4312.500 & 140264000.000 & 12902.503 \\
\hline \multicolumn{3}{|c|}{$\rho=3.3676 \times 10^{2} \mathrm{~g} / \mathrm{cm}^{3}$} \\
\hline 5.391 & 14545500.000 & 588.613 \\
\hline 8.215 & 14665000.000 & 591.546 \\
\hline 10.781 & 14723000.000 & 599.508 \\
\hline 15.682 & 14995000.000 & 602.836 \\
\hline 21.563 & 15179700.000 & 616.630 \\
\hline 34.500 & 15789200.000 & 636.221 \\
\hline 43.125 & 16093600.000 & 650.506 \\
\hline 86.250 & 17859500.000 & 726.080 \\
\hline 172.500 & 21530900.000 & 897.457 \\
\hline 345.000 & 30504600.000 & 1301.652 \\
\hline 690.000 & 50560500.000 & 2224.865 \\
\hline 1293.750 & 87731800.000 & 3952.434 \\
\hline 4312.500 & 281715000.000 & 12945.568 \\
\hline \multicolumn{3}{|c|}{$\rho=6.7352 \times 10^{2} \mathrm{~g} / \mathrm{cm}^{3}$} \\
\hline 5.391 & 47188700.000 & 985.291 \\
\hline 8.215 & 47374300.000 & 989.586 \\
\hline 10.781 & 47542300.000 & 993.478 \\
\hline 15.682 & 47866200.000 & 1000.964 \\
\hline 21.563 & 48258700.000 & 1010.034 \\
\hline
\end{tabular}

to be continued on next page 
TABLE I - continued from previous page

\begin{tabular}{|c|c|c|}
\hline Temperature(eV) & Pressure(GPa) & Internal energy (eV/atom) \\
\hline 34.500 & 49138100.000 & 1030.355 \\
\hline 43.125 & 49735000.000 & 1044.162 \\
\hline 86.250 & 52836300.000 & 1115.850 \\
\hline 172.500 & 59610600.000 & 1272.441 \\
\hline 345.000 & 75258300.000 & 1634.074 \\
\hline 690.000 & 112078000.000 & 2485.051 \\
\hline 1293.750 & 183785000.000 & 4142.350 \\
\hline 4312.500 & 566346000.000 & 12930.233 \\
\hline \multicolumn{3}{|c|}{$\rho=1.3470 \times 10^{3} \mathrm{~g} / \mathrm{cm}^{3}$} \\
\hline 5.391 & 152939000.000 & 1629.645 \\
\hline 8.215 & 153307000.000 & 1633.926 \\
\hline 10.781 & 153649000.000 & 1637.809 \\
\hline 15.682 & 152494000.000 & 1645.251 \\
\hline 21.563 & 155067000.000 & 1654.214 \\
\hline 34.500 & 156786000.000 & 1674.139 \\
\hline 43.125 & 157956000.000 & 1687.602 \\
\hline 86.250 & 163941000.000 & 1756.777 \\
\hline 172.500 & 176641000.000 & 1903.589 \\
\hline 345.000 & 204903000.000 & 2230.145 \\
\hline 690.000 & 271115000.000 & 2995.092 \\
\hline 1293.750 & 405010000.000 & 4541.812 \\
\hline 4312.500 & 1154030000.000 & 12958.110 \\
\hline
\end{tabular}

\section{A. Hugoniot curve}

High precision EOS data are essential for understanding target implosion process in ICF. We first examine the present DFT-EOS theoretically through the Rankine-Hugoniot (RH) 


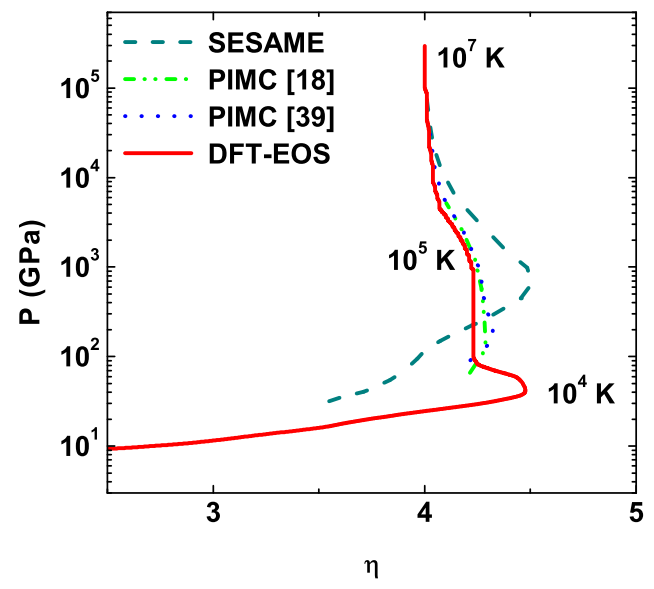

FIG. 3: (Color online) Present DFT-EOS for wide-range Hugoniot curve (red line) of liquid hydrogen. Previous wide-range Hugoniot curves from PIMC simulations (by S. X. Hu et al. [38] and Militzer et al. [18]) and SESAME [21] are also shown for comparison.

equations, which follow from conservation of mass, momentum, and energy across the front of the shock wave. The locus of points in $(E, P, V)$-space described by RH equations satisfy

$$
\begin{gathered}
E_{1}-E_{0}=\frac{1}{2}\left(P_{1}+P_{0}\right)\left(V_{1}-V_{0}\right), \\
\left(P_{1}-P_{0}\right)=\rho_{0} u_{s} u_{p}, \\
V_{1}=V_{0}\left(1-u_{p} / u_{s}\right),
\end{gathered}
$$

where subscripts 0 and 1 represent the initial and shocked state, and $E, P$, and $V$ denote internal energy, pressure, and volume, respectively. $u_{p}$ is the particle velocity of the material behind the shock front and $u_{s}$ is the shock velocity. Along the Hugoniot curve of the liquid hydrogen, the starting point with a density of $0.0855 \mathrm{~g} / \mathrm{cm}^{3}$ and a temperature of $23 \mathrm{~K}$ has been selected, where the relative internal energy has been set to zero and the pressure is considered as small as negligible. Smooth functions have been adopted to fit DFT-EOS in the relative density and temperature regime. Our DFT-based Hugoniot curve with pressure up to $10^{5} \mathrm{GPa}$ and temperature up to $5 \times 10^{7} \mathrm{~K}$ is presented in Fig. 3. Our simulation results indicate that the maximum shock compression ratio is 4.5 with a pressure around $40 \mathrm{GPa}$, at which the system is governed by gradual dissociation of molecules. With the increase of pressure, the compression ratio decreases and then reaches a value of 4.23 below $950 \mathrm{GPa}$. This hardening behavior of the Hugoniot can be attributed to the formation 
of mono-atomic fluid. However, as the pressure exceeds $10^{3} \mathrm{GPa}$ (temperature above 19 $\mathrm{eV}$ ), the compression ratio merges into 4.0, which indicate a full ionization of the liquid hydrogen. On the other side, SESAME Hugoniot [21] plotted in Fig. 3] shows a maximum compression ratio around 4.5, but the corresponding pressure is much too high with respect to our DFT-Hugoniot. For comparison, previous results from PIMC simulations [18, 38] are also plotted in Fig. 3, which shows consistency with our DFT results at pressures beyond 50 GPa. However, PIMC simulations have failed to reproduce the experimental results below $50 \mathrm{GPa}$.

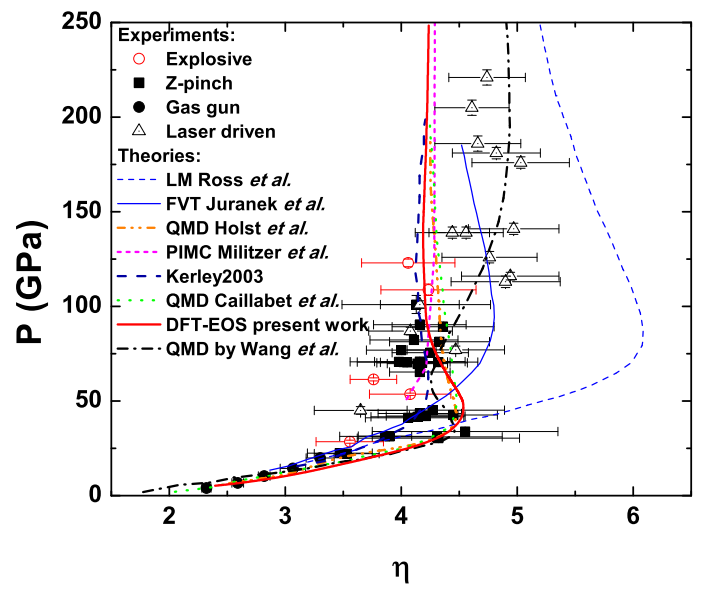

FIG. 4: (Color online) Hugoniot curve based on present DFT-EOS for liquid hydrogen with (red solid line) or without (red dashed line) considering ionic quantum zero-point energy. Previous experimental results and theoretical predictions are shown for comparison. Experimental data: gas gun by Nellis et al. [8] (solid circles), Z-pinch by Knudson et al. 10] (solid squares), explosives of Boriskov et al. [9] (open circles), laser-driven by Hicks et al. [13] (up open triangles), and Boehly et al. [12] (down open triangles). Theoretical data: QMD simulation results by Holst et al. (orange line) [39], Caillabet et al. (green line) [40], and Wang et al. (black dash-dotted line) [41], PIMC results by Militzer et al. (magenta line) [18], Kerley (royal line) [22], LM model by Ross (blue dashed line) [15], and FVT (blue solid line) [25].

Over the past ten years, the Hugoniot of hydrogen or deuterium has been experimentally explored up to $\sim 200 \mathrm{GPa}$. The latest set of data points were obtained by two-stage light gas gun [8], explosive-driven compression [9], Z-pinch-driven compression [10], where the compression ratio $\eta$ shows a maximum close to 4.3 , or by laser-driven compression with the Nova laser and the Omega laser (the EOS would possibly be corrected by introducing the 
quartz standard), which suggest a stiff behavior $\left(\eta_{\max } \approx 4.2\right)$ below $100 \mathrm{GPa}$ and become softer $\left(\eta_{\max } \approx 4.5 \sim 5.5\right)$ at higher pressures [12, 13]. To clearly show the comparison between the present DFT-EOS and those previous results, Fig. (4) plots the Hugoniot curve below 250 GPa. The present Hugoniot curve from DFT-EOS with accounting for the ionic quantum zero-point energy (ZPE) shows better accordance with experimental data. At pressures below $100 \mathrm{GPa}$, both of the curves, as discussed above, exhibit a maximum compression ratio of 4.5, which is accordant with previous QMD results and experimental data obtained by gas gun, converging explosives and magnetically driven flyer. However, as pressures go beyond $100 \mathrm{GPa}, \eta \sim 4.3$ indicates the agreement with high power laser experiments with the quartz standard. Predictions from various chemical models are also shown for comparison, for instance, Kerley 2003 [22], linear mixing model [15], and the fluid variational theory (FVT) [25]. Those chemical methods generally predict larger maximum compression ratio into higher pressures, except for the Kerley 2003 EOS, which is in better agreement with experiments.
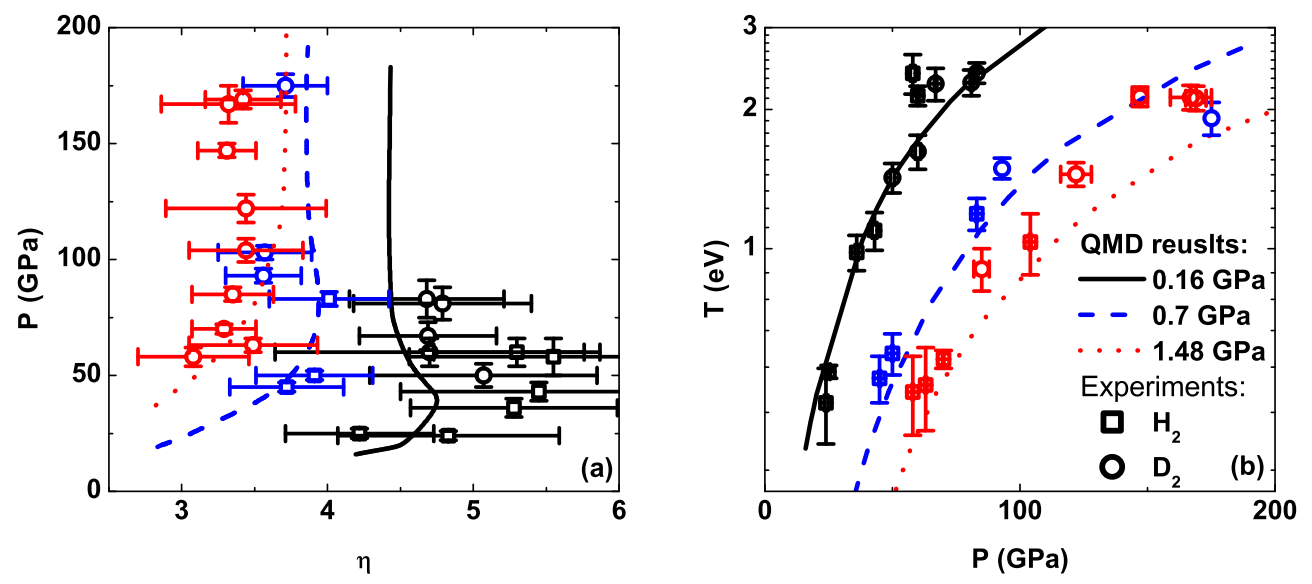

FIG. 5: (Color online) Pressure versus compression ratio (a) and temperature versus pressure (b). For comparison with the most recent experimental data [42], three initial pre-compressions of $\mathrm{H}_{2}$ $\left(\mathrm{D}_{2}\right)$ samples at $297 \mathrm{~K}$ have been studied: $0.16 \mathrm{GPa}$, $0.7 \mathrm{GPa}$, and $1.48 \mathrm{GPa}$.

Recently, laser-driven shock compressions on $\mathrm{H}_{2}$ or $\mathrm{D}_{2}$ precompressed in diamond anvil cells from 0.16 to $1.6 \mathrm{GPa}$ have been proved to provide visible ways to generate shock Hugoniot data over a significantly broader thermodynamical regime than previous experiments [42]. These experimental data are highly valuable for examining various theoretical models. In the present work, we have shown the Hugoniot data for initial pre-compressions of 0.16 
GPa, 0.7 GPa, and 1.48 GPa in Fig. 5. As a consequence, the data covers a density range $\sim 2$ times greater than previous investigations limited to the principal Hugoniot alone. As shown in Fig. 5, good agreement has been gained between the present DFT-EOS pre-compressed Hugoniot data and laser-driven experimental results. The maximum compression ratio along a given Hugoniot has been observed to strongly depend on the initial density. That is, with increasing initial density, the compression ratio decreases.

\section{B. Molecular dissociations}

For molecular fluid in the warm dense regime, which consists of atoms, molecules, nuclei, and electrons, the free energy can be expressed as

$$
F(\rho, T)=F_{i d}^{(i)}+F_{i d}^{(e)}+F_{e x}^{(i-e)}+F_{d i s}^{(m o l)},
$$

where $F_{i d}^{(i)}$ and $F_{i d}^{(e)}$ are the ideal free energies for ions and electrons, respectively, $F_{e x}^{(i-e)}$

is the excess free energy, while $F_{d i s}^{(m o l)}$ denotes the contribution from molecular dissociation with the following form [40]:

$$
F_{d i s}^{(m o l)}=N k_{B} T\left\{\ln \alpha+\frac{1-\alpha}{2}\right\} .
$$

Here the dissociation ratio $\alpha$ is used as an adjustable function of density and temperature, with an assumed Fermi-function form

$$
\alpha(\rho, T)=\frac{1}{\exp [B(\rho) / T-C(\rho) T]+1},
$$

where $B(\rho)=\exp \left(B_{1}+B_{2} \rho\right)$ and $C(\rho)=\exp \left(C_{1}+C_{2} \rho\right)$. Using the present DFT-EOS data, we have determined the value $B_{1}=9.5517, B_{2}=-2.8277, C_{1}=-8.2946$, and $C_{2}=0.4708$.

At temperatures below $10000 \mathrm{~K}$, molecular dissociation governs the first-order phase transition, which is important in determining the nonmetal-to-metal transition. In this work, we have introduced a Fermi formula to fit our DFT-EOS in warm dense region by using Eq. (11), and the dissociation fraction has been plotted in Fig. 6. Vorberger et al. [43] have introduced a criteria to estimate the fraction of molecular hydrogen by counting the number of atoms located within a radius, which last for a time greater than ten vibrational periods. Holst et al. [39] have used a coordination number,

$$
K(r)=\frac{N-1}{V} \int_{0}^{r} 4 \pi r^{\prime 2} g\left(r^{\prime}\right) d r^{\prime},
$$




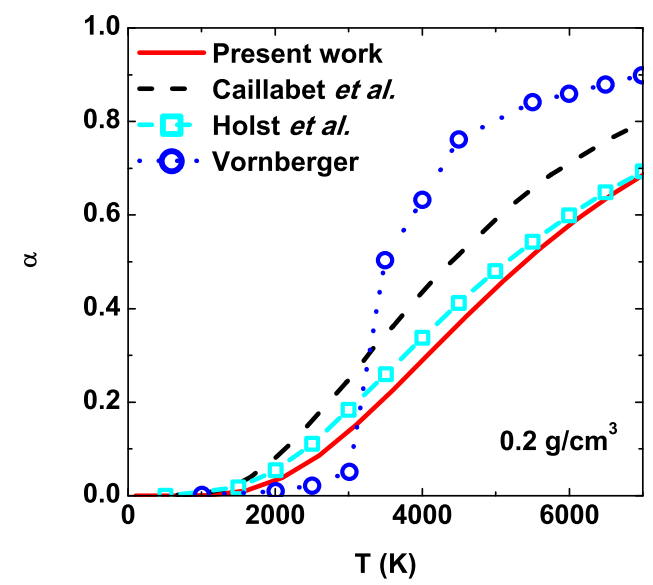

FIG. 6: (Color online) Calculated molecular dissociation fraction as a function of temperature at $0.2 \mathrm{~g} / \mathrm{cm}^{3}$. For comparison, previous theoretical results [39, 40, 43] have also been plotted.

to determine $\alpha$, where $g(r)$ is the pair correlation function to present the possibility of finding a particle from a reference atom. Results from those different assumptions are compared with the present work in Fig. 6. They yield similar tendency with temperature at the sampled density. The dissociation fraction from Vorberger et al. strongly depends on the definition of the molecule in this region, and shows abrupt increase as temperature increases. However, QMD method gives a smoother behavior of $\alpha$ as indicated in Fig. 6.

\section{Comparison of DFT-EOS with previous theoretical results}

In this section, the present DFT-EOS data have been systemically compared with previous theoretical predictions. At densities from $\sim 10^{-3} \mathrm{~g} / \mathrm{cm}^{3}$ to $\sim 10^{-1} \mathrm{~g} / \mathrm{cm}^{3}$, we have shown the pressure and internal energy difference between our DFT-EOS and those obtained by PIMC simulations (see Fig. 7). The results indicate a maximum of $7 \%$ difference for the pressure and $15 \%$ for the energy over the temperatures we explore. At temperatures above $\sim 30 \mathrm{eV}$, the distinction can be viewed as small as negligible between the two methods. In the warm dense regime, which is highlighted at densities between 0.2 and $3.0 \mathrm{~g} / \mathrm{cm}^{3}$, very good agreement has been found between our DFT-EOS data and those fitted QMD results at the temperature domain $2000 \sim 10000 \mathrm{~K}$ (left panel in Fig. 8). At temperature beyond $10000 \mathrm{~K}$, results from PIMC simulations by Militzer et al. [18], Chabrier Model [44], and QMD simulations [39, 40] have been plotted in the right panel in Fig. 8. The PIMC method 

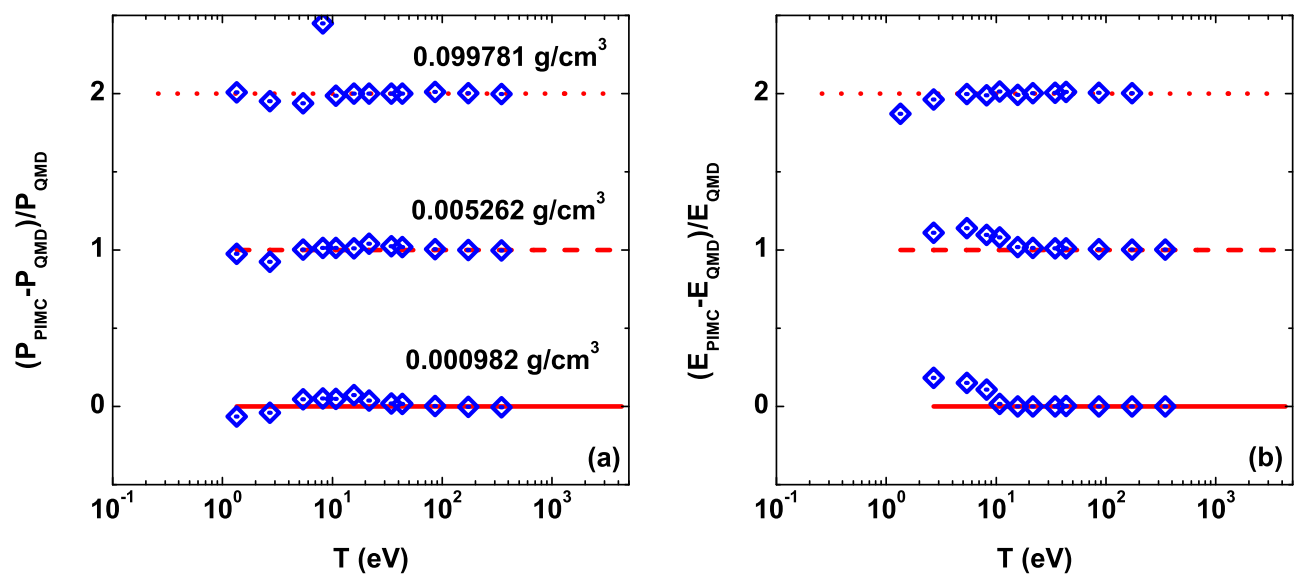

FIG. 7: (Color online) Pressure (a) and internal energy (b) differences between QMD and PIMC 26] methods as functions of temperature. QMD results are plotted as the red solid, red dashed, and red dotted lines at densities of $0.000982,0.005262$, and $0.099781 \mathrm{~g} / \mathrm{cm}^{3}$, respectively. The blue open diamonds denote PIMC data. Each curve corresponds to an isochore. Each curve has been shifted by 1.0 from the previous one for clarity.
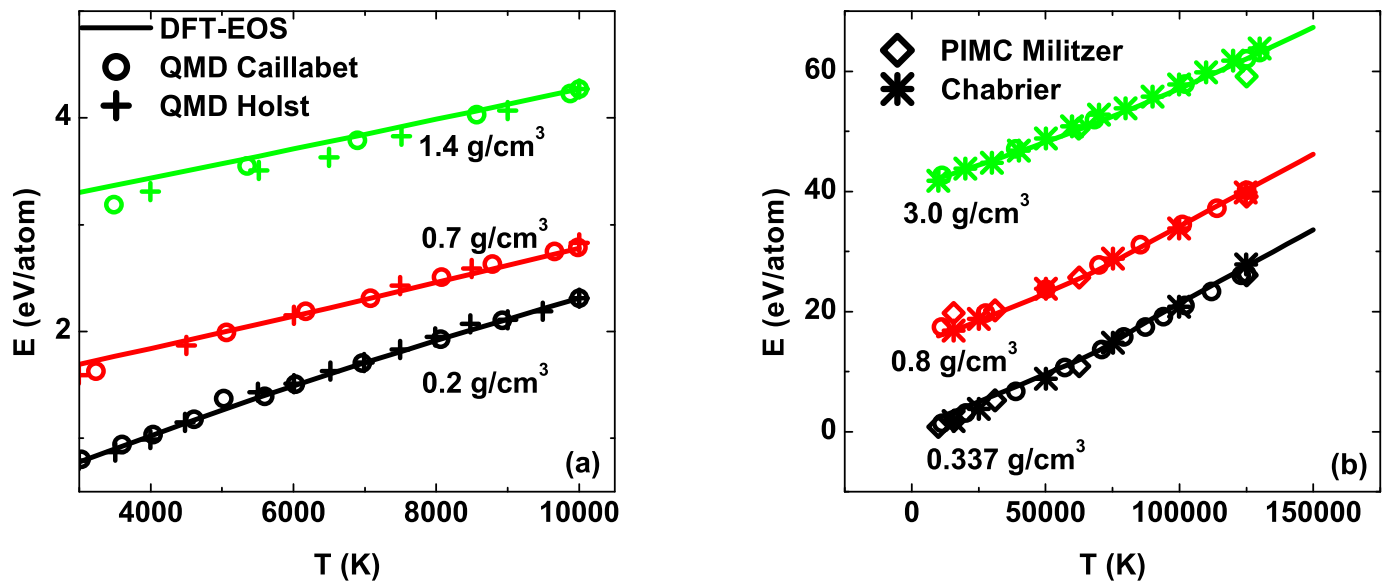

FIG. 8: (Color online) Internal energy of liquid hydrogen as a function of temperature. Previous QMD [39, 40], PIMC [18], and Chabrier model [44] results are also shown for comparison.

is suitable for investigating many-body quantum systems at high temperatures. In this method, electrons and ions are treated on equal footing as paths. The model of Chabrier and Potekhin considers a fully ionized plasma, which is reliable at high temperature and low density region. As shown in Fig. 8, the present results are in accordance with those numerical simulations and theoretical models. 

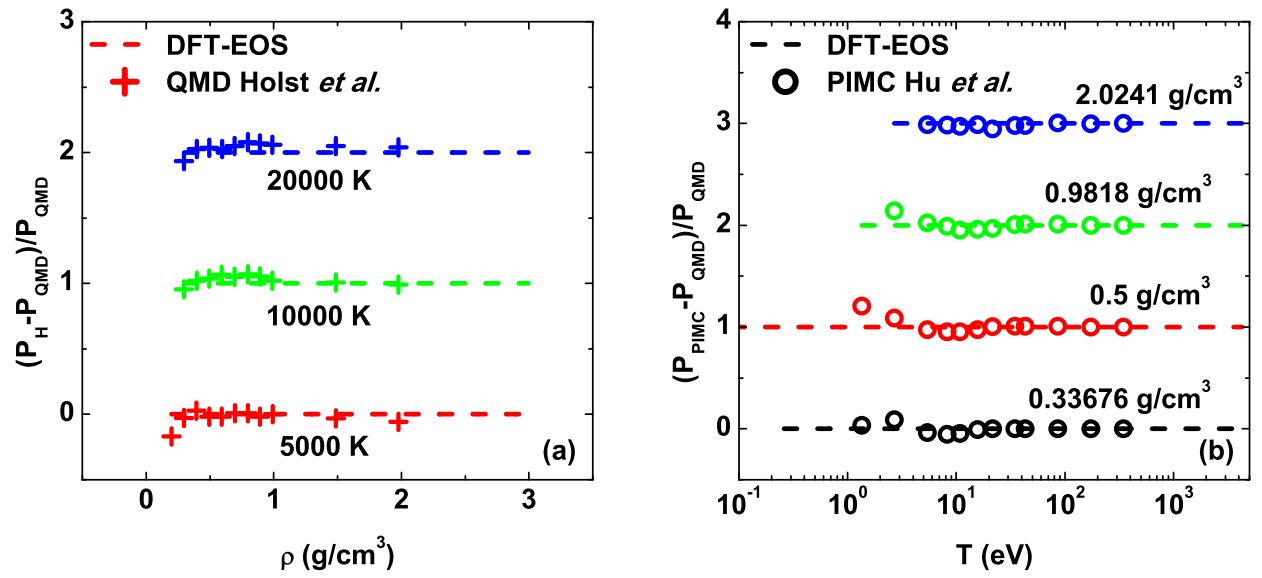

FIG. 9: (Color online) (a) Pressure difference as a function of density, $P_{\mathrm{H}}$ denotes data from Holst et al. [39]; (b) Pressure difference as a function of temperature, $P_{\text {PIMC }}$ are obtained from Hu et al. [26]. Each curve has been shifted by 1.0 from the previous one for clarity.
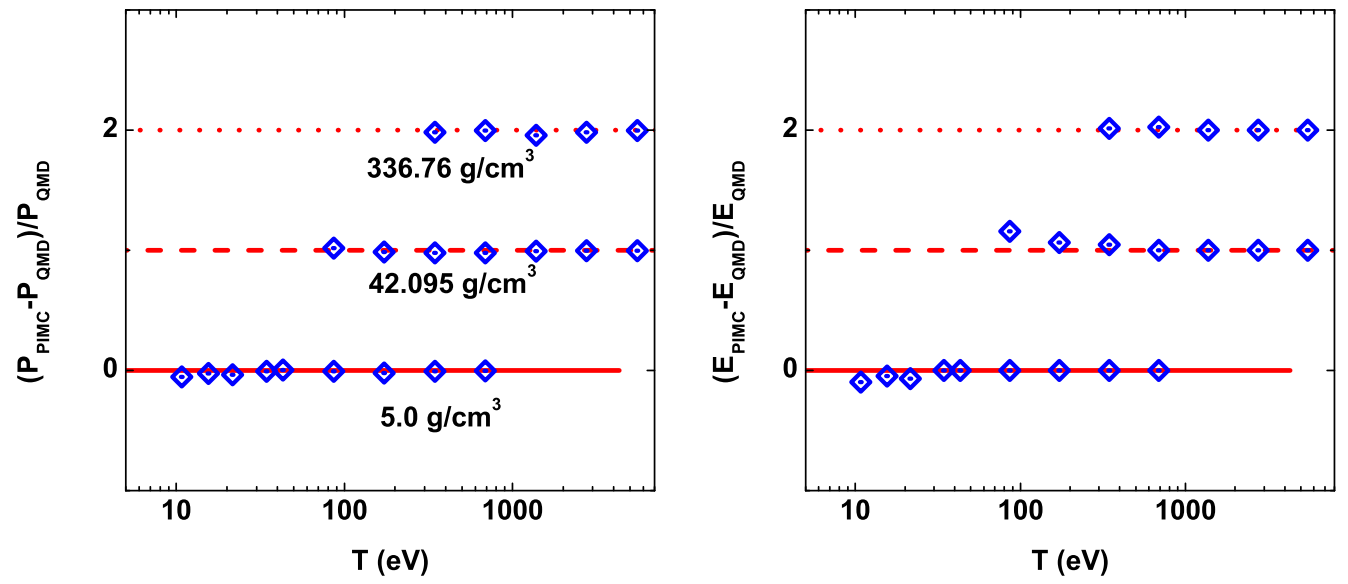

FIG. 10: (Color online) DFT-EOS is compared with PIMC data [26] in the dense plasma region. The present DFT-EOS results are shown as red lines, and blue open diamonds denote PIMC data. Each curve has been shifted by 1.0 from the previous one for clarity.

The isotherms of the pressure have been observed to show a systematic behavior in terms of the density and temperature (see Fig. 9). In this region, we do not find any signs for $\left(\frac{\partial P}{\partial V}\right)_{T}>0$, which would indicate another first-order phase transition (the so-called PPT). PPT is usually considered in chemical models such as fluid variational theory [25] or liquid state perturbation theory [45]. In these chemical models, minimization of the free energy for a mixture consists of atoms, molecules, and plasma in equilibrium. Relations 
between different particles are described by effective potentials. As we explore to a higher temperature region (right panels in Fig. 9 and Fig. 10]), PIMC data by $\mathrm{Hu}$ et al. [26] are shown for comparison. It is clearly indicated that the pressure given by DFT-EOS is in good agreement with PIMC calculations up to $\sim 10^{7} \mathrm{~K}$ for the densities concerned, and this agreement extends toward lower temperatures when the density decreases.

\section{CONCLUSION}

In summary, we have constructed a wide-range DFT-EOS by means of FPMD and OFMD simulations. After building short cutoff radius Columbic potential, we have the ability to explore the EOS into ultra-dense region. The present DFT-EOS is valid at densities from $9.82 \times 10^{-4}$ to $1.347 \times 10^{3} \mathrm{~g} / \mathrm{cm}^{3}$ with the temperature up to $5 \times 10^{7} \mathrm{~K}$. Available experimental data and theoretical models have been introduced to compare with current DFT-EOS. We have found good agreement between our results and those data probed by gas gun, chemical explosive, and magnetic driven plate flyer experiments, which indicate a maximum compression ratio of 4.5 around $40 \mathrm{GPa}$. At higher pressures, our data show stiff behavior and validates the high power laser experiments with quartz standard. The principal Hugoniot curve is also accordant with previous QMD simulation results. Agreement has also been found between our DFT-EOS Hugoniot data and those obtained by pre-compressed laserdriven shock wave experiments, which provide visible ways to generate EOS in a broader density and temperature regime. As density and temperature enter into a denser and hotter regime, where experimental detections are prohibited, the present results are compared with those predicted by chemical model and PIMC simulations. The present DFT-EOS covers typical states as can be reached in ICF and will be applied in hydrodynamic simulations in the future work.

\section{ACKNOWLEDGEMENT}

This work was supported by NSFC under Grants No. 11275032, No. 11005012 and No. 51071032, by the National Basic Security Research Program of China, and by the National High-Tech ICF Committee of China. 
[1] S. Atzeni and J. Meyer-ter-Vehn, The Physics of Inertial Fusion: Beam Plasma Interaction, Hydrodynamics, Hot Dense Matter, International Series of Monographs on Physics (Clarendon Press, Oxford, 2004).

[2] T. Guillot, Science 286, 72 (1999).

[3] T. Guillot, Planet. Space Sci. 47, 1183 (1999).

[4] R. L. McCrory, et al. Phys. Plasmas 15, 055503 (2008).

[5] J. D. Lindl, Phys. Plasmas 2, 3933 (1995).

[6] W. B. Hubbard, Science 214, 145 (1980).

[7] D. J. Stevenson, Annu. Rev. Earth Planet. Sci. 10, 257 (1982).

[8] W. J. Nellis, Rep. Prog. Phys. 69, 1479 (2006).

[9] G. V. Boriskov, A. I. Bykov, R. Ilkaev, V. D. Selemir, G. V. Simakov, R. F. Trunin, V. D. Urlin, A. N. Shuikin, and W. J. Nellis, Phys. Rev. B 71, 092104 (2005).

[10] M. D. Knudson, D. L. Hanson, J. E. Bailey, C. A. Hall, J. R. Asay, and C. Deeney, Phys. Rev. B 69, 144209 (2004).

[11] G. W. Collins, L. B. Da Silva, P. Celliers, D. M. Gold, M. E. Foord, R. J. Wallace, A. Ng, S. V. Weber, K. S. Budil, and R. Cauble, Science 281, 1178 (1998).

[12] T. R. Boehly, D. G. Hicks, P. M. Celliers, T. J. B. Collins, R. Earley, J. H. Eggert, D. JacobsPerkins, S. J. Moon, E. Vianello, D. D. Meyerhofer, and G. W. Collins, Phys. Plasmas 11, L49 (2004).

[13] D. G. Hicks, T. R. Boehly, P. M. Celliers, J. H. Eggert, S. J. Moon, D. D. Meyerhofer, and G. W. Collins, Phys. Rev. B 79, 014112 (2009).

[14] A. B. Belonoshko, A. Rosengren, N. V. Skorodumova, S. Bastea, and B. Johansson, J. Chem. Phys. 122, 124503 (2005).

[15] M. Ross, Phys. Rev. B 58, 669 (1998).

[16] H. Juranek and R. Redmer, J. Chem. Phys. 112, 3780 (2000).

[17] W. R. Magro, D. M. Ceperley, C. Pierleoni, and B. Bernu, Phys. Rev. Lett. 76, 1240 (1996).

[18] B. Militzer and D. M. Ceperley, Phys. Rev. Lett. 85, 1890 (2000).

[19] V. Bezkrovniy, V. S. Filinov, D. Kremp, M. Bonitz, M. Schlanges, W. D. Kraeft, P. R. Levashov, and V. E. Fortov, Phys. Rev. E 70, 057401 (2004). 
[20] T. Lenosky, S. Bickham, J. Kress, and L. Collins, Phys. Rev. B 61, 0163 (2000).

[21] G. I. Kerley, Phys. Earth Planet. Inter. 6, 78 (1972).

[22] G. I.Kerley, Sandia National Laboratory, Albuquerque,NM, Report SAND2003-3613 (2003).

[23] D. Saumon and G. Chabrier, Phys. Rev. A 46, 2084 (1992).

[24] F. J. Rogers, Contrib. Plasma Phys. 41, 179 (2001).

[25] H. Juranek, R. Redmer, and Y. Rosenfeld, J. Chem. Phys. 117, 1768 (2002).

[26] S. X. Hu, B. Militzer, V. N. Goncharov, and S. Skupsky, Phys. Rev. B 84, 224109 (2011).

[27] ABINIT (avaliable at www.abinit.org) is a common project of theUniversite Catholique de Louvain (Louvain-la Neuve, Belgium), Corning Incorporated, and other contributors.

[28] J. M. Haile, Molecular Dynamics Simulation (Wiley-Interscience, New York, 1997).

[29] F. Lambert, J. Clérouin, and S. Mazevet, Europhys. Lett. 75, 681 (2006).

[30] S. Mazevet, F. Lambert, F. Bottin, G. Zérah, and J. Clérouin, Phys. Rev. E 75, 056404 (2007).

[31] F. Lambert, J. Clérouin, J.-F. Danel, L. Kazandjian, and G. Zérah, Phys. Rev. E 77, 026402 (2008).

[32] M. Brack and R. K. Bhaduri, Semiclassical Physics (Westview, Oxford, 2003).

[33] F. Perrot, Phys. Rev. A 20, 586 (1979).

[34] C. Wang, X.-T. He, and P. Zhang, Phys. Rev. Lett. 106, 145002 (2011).

[35] C. Wang, X.-T. He, and P. Zhang, Phys. Plasmas 19, 042702 (2012).

[36] S. Goedecker, M. Teter, J. Huetter, Phys. Rev. B 54, 1703 (1996).

[37] The time steps have been taken as $\Delta t=a / 20 \sqrt{k_{B} T / m_{H}}$, where $a=\left(3 / 4 \pi n_{i}\right)^{1 / 3}$ is the ionic sphere radius ( $n_{i}$ is the ionic number density), $k_{B} T$ presents the kinetic energy, and $m_{H}$ is the ionic hydrogen.

[38] S. X. Hu, B. Militzer, V. N. Goncharov, and S. Skupsky, Phys. Rev. Lett. 104, 235003 (2010).

[39] B. Holst, R. Redmer, and M. P. Desjarlais, Phys. Rev. B 77, 184201 (2008).

[40] L. Caillabet, S. Mazevet, and P. Loubeyre, Phys. Rev. B 83, 094101 (2011).

[41] C. Wang, X.-T. He, and P. Zhang, J. Appl. Phys. 108, 044909 (2010).

[42] P. Loubeyre, S. Btygoo, J. Eggert, P. M. Celliers, D. K. Spaulding, J. R. Rygg, T. R. Boehly, G. W. Collins, and R. Jeanloz, Phys. Rev. B 86, 144115 (2012).

[43] J. Vorberger, I. Tamblyn, B. Militzer, and S. A. Bonev, Phys. Rev. B 75, 024206 (2007).

[44] G. Chabrier and A. Y. Potekhin, Phys. Rev. E 58, 4941 (1998).

[45] D. Saumon, G. Chabrier, and H. M. van Horn, Astrophys. J. Suppl. Ser. 99, 713 (1995). 